\title{
The star formation histories of Low Surface Brightness galaxies
}

\author{
E. F. Bell, R. G. Bower, R. S. de Jong, B. J. Rauscher \\ Department of Physics, University of Durham, Science Laboratories, \\ South Road, Durham DH1 3LE, UK \\ D. Barnaby, D. A. Harper, Jr., M. Hereld, R. F. Loewenstein \\ Department of Astronomy and Astrophysics, University of Chicago, \\ 5640 S. Ellis Ave., Chicago, IL 60637, USA
}

\begin{abstract}
Near-infrared images of a sample of red, blue and giant low surface brightness disk galaxies (LSBGs) were combined with optical data with the aim of constraining their star formation histories. Most LSBGs have strong colour gradients consistent with mean stellar age gradients. We find that LSBGs have a large range of ages and metallicities, spanning those observed in normal disk galaxies. In particular, red and blue LSBGs have very different star formation histories and represent two independent routes to low $B$ band surface brightness. Blue LSBGs are well described by models with low, roughly constant star formation rates, whereas red LSBGs are better described by a 'faded disk' scenario.
\end{abstract}

\section{Introduction}

There has been much recent debate on the star formation histories of low surface brightness disk galaxies (LSBGs; galaxies with $B$ band central surface brightnesses fainter than 22.5 mag arcsec ${ }^{-2}$ ). The best studied LSBGs are blue (McGaugh \& Bothun 1994; de Blok, van der Hulst \& Bothun 1995), indicating a young mean stellar age and/or low metallicity. Their measured H II region metallicities are low, at around or below 1/3 solar abundance (McGaugh 1994; Rönnback \& Bergvall 1995; de Blok 1997). Morphologically, the best studied LSBGs appear to have disks, but little spiral structure (McGaugh, Schombert $\&$ Bothun 1995). The massive star formation rates (SFRs) in LSBGs are an order of magnitude lower than those of high surface brightness (HSB) galaxies (van der Hulst et al. 1993). H I observations show that LSBGs have high gas mass fractions, sometimes even approaching unity (de Blok, McGaugh \& van der Hulst 1996; McGaugh \& de Blok 1997). As yet, there have been no CO detections of LSBGs, only upper limits on the $\mathrm{CO}$ abundances which indicate that LSBGs have CO/H I ratios significantly lower than those of HSB galaxies (Schombert et al. 1990; de Blok 1997). These observations are consistent with a scenario in which LSBGs are relatively unevolved, low mass surface density, low metallicity systems, with roughly constant or even increasing SFRs (de Blok 1997). 
This scenario has been called into question however by the recent discovery of a population of red LSBGs (O'Neil, Bothun \& Cornell 1997; O'Neil et al. 1997). The optical colours of these galaxies are similar to those of old stellar populations, but the red colours could be caused by age or metallicity effects. The same age-metallicity degeneracy plagues the analysis of the colours of blue LSBGs. Padoan, Jiminez and Antonuccio-Delogu (1997) question the apparent youth of blue LSBG stellar populations: they find that their optical colours are consistent with those of old, very low metallicity stellar populations.

Another puzzle is posed by the results of Quillen and Pickering (1997) who recently obtained near-infrared (NIR) $H$ band imaging of two LSBG giants (galaxies similar to but less extreme than Malin 1). They concluded that the optical-NIR colours of both the central and outer regions of their galaxies were compatible with those seen in old stellar populations (such as E/S0 galaxies). This is difficult to understand, especially in the context of the relatively high gas fractions and low (but non-negligible) star formation rates observed in giant LSBGs.

Here, we explore the star formation histories (SFHs) of different types of LSBG. Because of the age/metallicity degeneracy inherent in optical broad-band colours, it is impossible to tell, using optical colours alone, what causes the colour differences between these classes of LSBG, and what drives their optical colour gradients (de Blok, van der Hulst \& Bothun 1995). NIR images, in conjunction with optical data, offer the first chance to break this degeneracy, and constrain plausible SFHs.

\section{Observations and data reduction}

Our sample consists of galaxies in both the northern and southern hemisphere. The northern hemisphere sample, imaged in the near-infrared $K^{\prime}$ passband $(1.94-2.29 \mu \mathrm{m})$ using the Apache Point Observatory $3.5-\mathrm{m}$, is taken from de Blok, McGaugh \& van der Hulst (1996), O'Neil, Bothun \& Cornell (1997) and Sprayberry et al. (1995) and is selected to have estimated $22.5 \leq \mu_{B, 0} \leq 23.5$ mag $\operatorname{arcsec}^{-2}$ and $R_{25} \geq 16$ arcsec where $\mu_{B, 0}$ denotes the $\mathrm{B}$ band intrinsic disc central surface brightness, and $R_{25}$ denotes the major axis radius to the $25 \mathrm{~B} \mathrm{mag} \mathrm{arcsec}-2$ isophote. The pixel scale is $0.473 \mathrm{arcsec} / \mathrm{pixel}$, and typical on-source exposure times range between 15 and 25 minutes. Our southern hemisphere sample, imaged in the near-infrared $K_{d a r k}$ passband $(2.27-2.45 \mu \mathrm{m})$ using the South Pole 0.6-m, is selected from the ESO-Uppsula Catalogue (Lauberts \& Valentijn 1987) to have larger sizes and lower surface brightnesses: $\mu_{B, 0} \geq 23.0$ mag arcsec ${ }^{-2}, 65 \operatorname{arcsec} \leq R_{e f f} \leq 150$ arcsec and inclination less than $67^{\circ}$ (where $\mathrm{R}_{e f f}$ denotes the half-light radius). The pixel scale is $4.2 \mathrm{arcsec} / \mathrm{pixel}$, and typical on-source exposure times range between 3.5 and 7.5 hours. We also require $B$ and $R$ optical images for our analysis. We use existing data from the source papers where available, and have acquired $B$ and $R$ images for those galaxies without existing optical data. Further details of our sample, data acquisition and reduction will be presented in Bell et al. (1999); details for a subset of five northern hemisphere galaxies are presented in Bell et al. (1998). Our sample is by no means complete, but instead is meant to explore the range of disk LSBG star formation histories. 


\section{Results}

Surface photometry was carried out using the IRAF task ellipse. The centroid of the brightest region of the galaxy in $R$ band is taken to be the galaxy centre. The galaxy ellipticity and position angle were determined from the $R$ band outermost isophotes. Due to the low surface brightness of our sample in all passbands, the error in the sky level dominates the uncertainty in the photometry. Estimation of the sky level involves both the mean values in the outermost annuli of the surface photometry and the mean sky level in areas of the image that were free of galaxy emission and contamination from starlight. This sky level, which is an average over large areas, is typically accurate to a few parts in $10^{5}$ for the $K^{\prime}$ images, and better than $\sim 0.4$ per cent for the $K_{\text {dark }}$ and optical images. Galactic extinction corrections are from Schlegel, Finkbeiner \& Davis (1998), and range between 0.07 and $0.20 \mathrm{mag}$ in the $B$ band. K-corrections are applied to our two LSBG giants using non-evolving Sbc spectra from King \& Ellis (1985), and are typically $0.2 \mathrm{mag}$ in $B, 0.02 \mathrm{mag}$ in $R$ and $-0.2 \mathrm{mag}$ in $K$. Our findings are robust to reasonable uncertainties in the assumed K-correction. Galactic extinction corrected galaxy colours in three radial bins $(0<r / h<0.5,0.5<r / h<1.5$ and $1.5<r / h<2.5$ where $h$ is the $R$ band disk scale length) using images degraded to the same angular resolution are shown in Fig. 1.

It is clear that there are colour gradients in our sample of LSBGs. However, in order to interpret the colour gradients, it is necessary to compare the data with the results of a stellar population synthesis code, such as that of Bruzual \& Charlot (1998). In Fig. 1, we use single metallicity stellar populations with a Salpeter (1955) IMF and a star formation rate described by an exponential star formation timescale $\tau$. Note that the effects of chemical evolution are not included in this model. The solid lines represent the colours of stellar populations with a fixed metallicity and a range of star-forming timescales from an instantaneous burst to a constant SFR. The dashed lines represent the colours produced with a given star formation timescale and a range of metallicities. The arrow denotes the dust reddening vector given by a screen model using the extinction curve of Rieke \& Lebofsky (1985) for a visual extinction of $0.3 \mathrm{mag}$. There is some uncertainty in the placement of the model grid. Charlot, Worthey \& Bressan (1996) discussed the sources of error in stellar population synthesis models, and concluded that the uncertainty in model calibration is about 0.08 mag in $B-R$ colour, and around $0.13 \mathrm{mag}$ in $R-K$ colour, which is comparable to the calibration error bar in Fig. 1.

The observed colour gradients are consistent with a mean stellar age gradient (parameterised by the exponential star forming timescale $\tau$ ), along with an expected contribution from metallicity effects. Note that we can rule out any strong metallicity gradients in our sample ( $~ 0.5$ dex per disk scale length). The colour gradients may also have a contribution from the effects of differential dust reddening: this is expected to be a small effect however and is discussed further in the next section. The existence of these colour gradients is insensitive to any zero point uncertainties, and are very robust to the maximum flat fielding and sky level uncertainties.

It is clear from Fig. 1 that LSBGs span a wide range of luminosity-weighted stellar ages and metallicities, indicating a wide diversity in their inferred star formation histories. Red LSBGs appear to be older and more metal-rich than their 


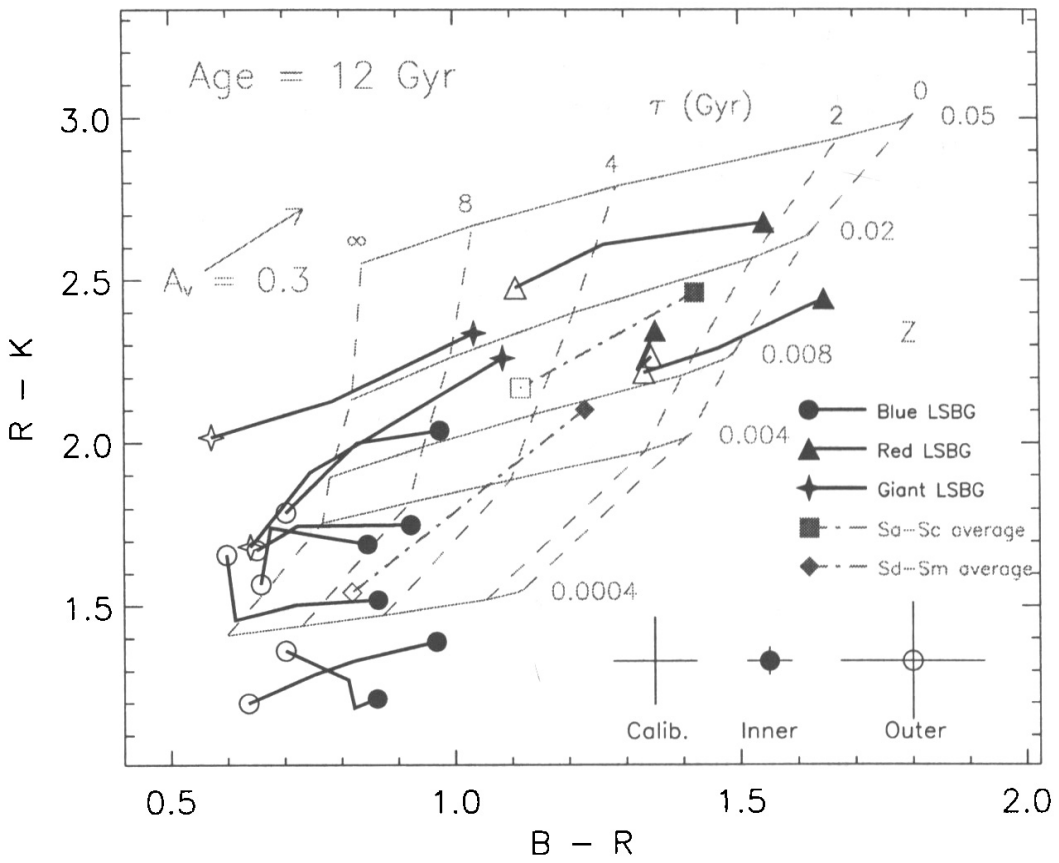

Figure 1. A colour-colour plot comparing the different stellar populations in our LSBG sample. The solid symbols denote central colours, and the open symbols the colours at 2 disk scale lengths. The solid lines are for our sample of LSBGs, where symbol style denotes galaxy type: circles are blue LSBGs, triangles are red LSBGs, and 4-pointed stars are giant LSBGs. Dot-dashed lines connect average colours of $\mathrm{Sa}-\mathrm{Sc}$ (squares) and $\mathrm{Sd}-\mathrm{Sm}$ (diamonds) galaxies from the sample of de Jong (1996). Typical colour errors caused by sky level uncertainty are shown by the error bars in the lower right hand corner, where the central colour errors are denoted by the solid circle and those at 2 disk scale lengths are denoted by the open circle. The calibration uncertainties are also shown. The model grid, based on the stellar population synthesis models of Bruzual and Charlot (1998), is discussed in the text. 
blue counterparts, indicating an epoch of more vigorous star formation. Blue LSBGs appear to be consistent with models with low, roughly constant star formation rates (implying young luminosity-weighted ages) and low stellar metallicities. Giant LSBGs are metal-rich, but appear to have significant younger stellar populations. Note that the stellar metallicities, and stellar metallicity differences between galaxy types, are consistent with the results of Hil region spectroscopy, where available (McGaugh 1994; Rönnback \& Bergvall 1995; de Blok 1997). Also, for the blue LSBGs, the optical and NIR colours of our galaxies imply stellar metallicities typically a factor of 20 higher than those proposed by Padoan, Jiminez and Antonuccio-Delogu (1997), thus ruling out their proposed SFH. Our conclusions are unchanged if other combinations of optical-NIR colours, or other stellar population synthesis codes, are used in the analysis.

\section{Discussion}

\subsection{Are the stellar population differences real?}

The differences between blue and red LSBGs, and the colour gradients, look as if they may be consistent with the effects of differing amounts of dust reddening in a foreground screen. However, the lack of strong dust features in optical LSBG images (McGaugh \& Bothun 1994; de Blok, van der Hulst \& Bothun $1995)$ and generally low ( $A_{B} \sim 1 \mathrm{mag}$ ) Balmer decrements towards LSBG H II regions (McGaugh 1994) tend to argue against large amounts of dust in LSBGs. Furthermore, when more realistic mixtures of dust and stars are used, it is found that the reddening vector steepens considerably, and that larger amounts of dust (typically a few times more) are required to produce the same displacements on a colour-colour plot (de Jong 1996). It is therefore unlikely that the effects of differential dust reddening dominate the colour trends in our sample.

Uncertainties in the high-mass end of the stellar IMF do not alter these conclusions. The above analysis assumes a Salpeter (1955) IMF, however, use of a Scalo (1986) or Miller \& Scalo (1979) IMF only significantly changes the high metallicity, large $\tau$ end of the grid. While this could change the absolute interpretation of colour gradients in terms of values of metallicity and $\tau$, it is still possible to spot relative metallicity and age trends in and between galaxies, and remain largely unaffected by this uncertainty. In fact, this serves to illustrate the inherent uncertainty in using broad band colours to attempt to unambiguously determine the star formation history of galaxies.

\subsection{Do red and blue LSBGs share common ancestors?}

Fig. 1 suggests that the red LSBGs have undergone a period of more vigorous star formation (both from the dominance of the older stellar populations, and from the inferred stellar metallicities), whereas blue LSBGs are well described by stellar populations with ongoing low metallicity star formation. However, is it possible that the red and blue LSBGs share the same origin: that is, can red and blue LSBGs transform from one into the other readily? In Fig. 2, we address this question.

Our red LSBGs, using the models of Bruzual \& Charlot (1998), have the optical-NIR colours of an old stellar population with roughly solar metallicity. If 


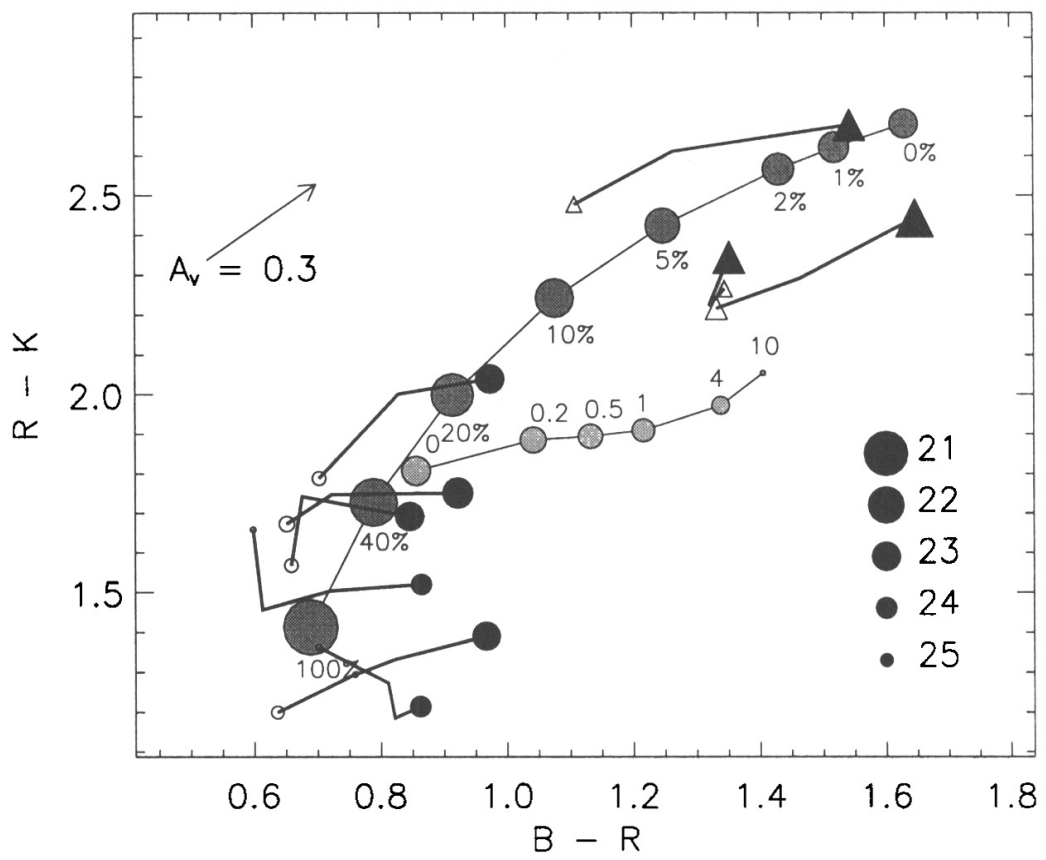

Figure 2. A plot showing the possibilities for transformation between red and blue LSBGs. Central and outer colours for our sample are denoted by solid and empty dark symbols (triangles for red LSBGs and circles for blue LSBGs), with their sizes indicating the $B$ band surface brightnesses at those radii. The upper track (dark grey) shows the colours of a $12 \mathrm{Gyr}$ old solar metallicity stellar population. Added to it is a 1.25 Gyr old single burst population with $Z=0.004$ with mass fractions ranging from 0 to 100 per cent. The lower track (light grey) shows colours of a $\tau=16 \mathrm{Gyr}$ model with an age of $12 \mathrm{Gyr}$, with the star formation truncated between 0 and 10 Gyr ago. Plot symbol size denotes changes in $B$ band surface brightness in mag arcsec ${ }^{-2}$. 
one adds 20 to 30 per cent, by mass, of a young $\sim 1$ Gyr stellar population with low metallicity $Z \lesssim 0.004$ (dark grey filled circles) it is possible to reproduce the colours of the blue LSBGs. However, the addition of young stars at all radii (to reproduce the colour of blue LSBGs at all radii) will increase the blue surface brightness by $\sim-2 \mathrm{mag}$, giving central surface brightnesses of $\sim 21 \mathrm{mag} \mathrm{arcsec}^{-2}$ for these 'transformed' red LSBGs. Thus, it is impossible to transform a red LSBG into a blue LSBG due to surface brightness constraints.

Alternatively, to transform a blue LSBG into a red one, the SFH must be truncated (light grey filled circles). Truncation of star formation will cause the optical colours to redden (note however that the stellar metallicity would still appear lower than those observed in our red LSBGs) at the expense of surface brightness (dimming of $\sim 1.5 \mathrm{mag}$ in $B$, and $\sim 0.8 \mathrm{mag}$ in $K$ is expected after 4 Gyr, when compared to a similar galaxy at the time of truncation which is still forming stars with $\tau \sim 16 \mathrm{Gyr}$ ). Therefore, blue LSBGs cannot reproduce our sample of red LSBGs because of metallicity constraints, and because the surface brightness would be too faint. Fading from an approximately solar metallicity HSB galaxy to a red LSBG would be possible. Note that an IMF heavily biased towards low-mass stars would also show similar colours, as the optical-NIR colours in essence indicate that red LSBGs simply lack high-mass stars.

\section{Conclusions}

As part of an ongoing study into the stellar populations of LSBGs, we obtained NIR images of a number of LSBGs spanning a wide range of star formation properties. With the addition of optical $B$ and $R$ images, we found the following.

- Optical-NIR radial colour gradients are common in LSBGs and are consistent with mean stellar age gradients, with the outer regions of LSBGs being younger than the central regions.

- Red LSBGs have the optical-NIR colours of old roughly solar metallicity stellar populations. In contrast, blue LSBGs are still actively forming stars, albeit at a low overall rate (van der Hulst et al. 1993), inconsistent with the SFH proposed by Padoan, Jiminez \& Antonuccio-Delogu (1997). LSBG giants have roughly solar metallicity, but have significant contributions from young stellar populations.

- These results suggest that red and blue LSBGs have very different star forming histories, and represent two independent routes to low $B$ band surface brightness: the blue LSBGs are well described by models with a low, roughly constant SFRs, whereas the red LSBGs are more consistent with a 'faded HSB disk' scenario.

Acknowledgments. We would like to thank Erwin de Blok, Stacy McGaugh, Karen O'Neil and David Sprayberry for providing surface photometry and images of galaxies in their sample, and for helpful discussions. In particular, we would like to thank Karen O'Neil for providing the coordinates of her LSBG sample before their publication. We also thank the U. Chicago TAC for regular time allocations to make the $K^{\prime}$ observations. Some of the observations 
described here were made during service time at the Isaac Newton Telescope and at the United Kingdom Infrared Telescope.

\section{References}

Bell, E. F., Bower, R. G., de Jong, R. S., Hereld, M. \& Rauscher, B. J. 1998, submitted to MNRAS

Bell, E. F., Barnaby, D., Rauscher, B. J., Bower, R. G., de Jong, R. S., Harper, Jr., D. A., Hereld, M. \& Loewenstein, R. F. 1999, in preparation

Bruzual, A. G. \& Charlot, S. 1998, in preparation

Charlot, S., Worthey, G. \& Bressan, A. 1996, ApJ, 457, 625

de Blok, W. J. G. 1997 PhD thesis, Groningen University

de Blok, W. J. G., McGaugh, S. S. \& van der Hulst, J. M. 1996, MNRAS, 283, 18

de Blok, W. J. G., van der Hulst, J. M. \& Bothun, G. D. 1995, MNRAS, 274, 235

de Jong, R. S. 1996, A\&A, 313, 377

King, C. R. \& Ellis, R. S. 1985, ApJ, 288, 456

Lauberts, A. \& Valentijn, E. A. 1989, The Surface Photometry Catalogue of the ESO-Uppsula Catalogue

McGaugh, S. S. 1994, ApJ, 426, 135

McGaugh, S. S. \& Bothun, G. D. 1994, AJ, 107, 530

McGaugh, S. S., Schombert, J. M. \& Bothun, G. D. 1995, AJ, 109, 2019

McGaugh, S. S. \& de Blok, W. J. G. 1997, ApJ, 481, 689

Miller, G. E. \& Scalo, J. M. 1979, ApJS, 41, 513

O'Neil, K., Bothun, G. D. \& Cornell, M.E. 1997, AJ, 113, 1212

O'Neil, K., Bothun, G. D., Schombert, J. M., Cornell, M. E. \&. Impey, C. D. 1997, AJ, 114, 2448

Padoan, P., Jiminez, R. \& Antonuccio-Delogu, V. 1997, ApJ, 481, L27

Quillen, A. C. \& Pickering, T. E. 1997, astro-ph/9705115

Rieke, G. H. \& Lebofsky, M. J. 1985, ApJ, 288, 618

Rönnback, J. \& Bergvall, N. 1995, A\&A, 302, 353

Salpeter, E. E. 1955, ApJ, 121, 61

Scalo, J. M. 1986, Fundam. Cosmic Phys., 11, 1

Schlegel, D. J., Finkbeiner, D. P. \& Davis, M. 1998, ApJ, 500, 525

Schombert, J. M., Bothun, G. D., Impey, C. D. \& Mundy, L. G. 1990, AJ, 100, 1523

Sprayberry, D., Impey, C. D., Bothun, G. D. \& Irwin, M. J. 1995, ApJ, 438, 72 van der Hulst, J. M., Skillman, E. D., Smith, T. R., Bothun, G. D., McGaugh, S. S. \& de Blok, W. J. G. 1993, AJ, 106, 548 